\title{
Early problematic eating behaviours are associated with lower fruit and vegetable intake and less dietary variety at 4-5 years of age. A prospective analysis of three European birth cohorts
}

\author{
A. Oliveira ${ }^{1,2, *}$, L. Jones ${ }^{3}$, B. de Lauzon-Guillain ${ }^{4,5}$, P. Emmett ${ }^{3}$, P. Moreira ${ }^{2,6}$, M. A. Charles ${ }^{4,5}$ \\ and C. Lopes ${ }^{1,2}$ \\ ${ }^{1}$ Department of Clinical Epidemiology, Predictive Medicine and Public Health, University of Porto Medical School, \\ Alameda Professor Hernâni Monteiro, 4200-319 Porto, Portugal \\ ${ }^{2}$ EPIUnit - Institute of Public Health, University of Porto, Rua das Taipas 135-139, 4050-600 Porto, Portugal \\ ${ }^{3}$ School of Social and Community Medicine, University of Bristol, Oakfield Grove, Bristol BS8 2BN, UK \\ ${ }^{4}$ INSERM, UMR1153 Epidemiology and Biostatistics Sorbonne Paris Cité Center (CRESS), Developmental Origins of Health \\ and Disease (ORCHAD) Team, F-94807 Villejuif, France \\ ${ }^{5}$ Paris Descartes University, Rue de l'Ecole de Médecine 75270, Paris Cedex 06, France \\ ${ }^{6}$ Faculty of Nutrition and Food Sciences, University of Porto, Rua Dr. Roberto Frias, 4200-465 Porto, Portugal
}

(Submitted 29 September 2014 - Final revision received 23 April 2015 - Accepted 1 June 2015 - First published online 21 July 2015)

Problematic eating behaviours during early childhood could be mediators of poor dietary habits. This study aims to prospectively relate early eating behaviours with fruit and vegetable (F\&V) intake and a healthy diet variety score of children aged between 4 and 5 years. Eating behaviours were assessed in three European birth cohorts (Generation XXI from Portugal, ALSPAC from the UK and EDEN from France) at 4-6, 12-15, 24 and 48-54 months of age, based on the child's feeding difficulties, mother's perception of child's poor eating (eating small quantities at each meal, not eating enough or needing to be stimulated to eat), food refusal and difficulties in the establishment of daily food routines. Daily servings of $\mathrm{F} \& \mathrm{~V}$ ( $>1 v$. $\leq 1$ serving/d, except in Generation XXI: $>3 v . \leq 3$ ) and the Healthy Plate Variety Score (categorised by the median score of each sample) were calculated using FFQ. Associations were tested by logistic regressions adjusted for maternal age, education, smoking during pregnancy, any breast-feeding and the child's $z$-score BMI at $4-5$ years of age. Children with more feeding difficulties, poor eating, food refusal/neophobia and difficulties in establishing a daily routine at 12-15, 24 and 48-54 months of age had in general lower F\&V intake at 4-5 years of age. The association with vegetables was slightly stronger than with fruits. These early feeding problems were also inversely associated with the variety score at 4-5 years of age, particularly when eating behaviours were reported after 12-15 months of age. A better understanding of these early feeding difficulties may help define strategies to increase the dietary quality in children.

Key words: Feeding behaviour: Fruit and vegetables: Diet variety: Cohort studies: Pre-school children

As infants and toddlers grow up, some problematic eating behaviours perceived by caregivers may develop ${ }^{(1-3)}$. Children who display difficulties in eating or reject certain types of foods that parents think are appropriate are often called problem feeders, picky eaters or neophobics. Children with food neophobia are reluctant to eat new foods, whereas picky children resist eating many familiar (as well as unfamiliar) foods ${ }^{(4)}$; both have been frequently associated with eating small meals, eating slowly and accepting a limited number of foods ${ }^{(5,6)}$. Thus, children displaying these feeding problems are likely to avoid particular foods or food groups, which could result in a limited food selection and lack of dietary variety, and have a negative impact on their intake of essential nutrients.

Feeding problems are considered as problems that arise during infancy and early childhood related with failure of the child's feeding learning process ${ }^{(2)}$. Aldridge et al. ${ }^{(2)}$ describes that there are specific risk periods of adaptations in feeding style and dietary intake, such as the introduction of complementary feeding and the self-feeding period. Most infants overcome the typical difficulties of these risky periods and their feeding style matures through practice and repeated exposures to new experiences, but some children fail to develop the skills

Abbreviations: F\&V, fruit and vegetable; HPVS, Healthy Plate Variety Score.

* Corresponding author: A. Oliveira, email acmatos@med.up.pt 
necessary to cope with developmental demands and face persistent problems with eating ${ }^{(2)}$.

Previous studies have shown that feeding problems are common in healthy young children and may compromise their future health - namely, determining a worse weight status ${ }^{(7,8)}$, increasing the likelihood of behavioural problems during later childhood $^{(9,10)}$ and symptoms of anorexia nervosa during adolescence $^{(11)}$. Previous research has also documented a negative effect of feeding problems on dietary intake ${ }^{(7,8,12-17)}$ and dietary variety ${ }^{(5,18,19)}$, overall suggesting that neophobia and pickiness are both associated with less healthy food choices in children. These associations have been mainly documented in relation to the consumption of specific food groups, such as fruits and/or vegetables and protein foods ${ }^{(7,8,12-17,19)}$ or specific nutrients ${ }^{(7,8,19)}$. Fewer studies have documented their relation with dietary variety ${ }^{(5,18,19)}$, and most of them have been conducted in children older than 5 years of age ${ }^{(5,19)}$ or have defined dietary variety based on a single question ${ }^{(5)}$.

Although there is previous evidence that certain early eating behaviours may compromise later dietary intake and variety, most of the studies conducted so far are cross-sectional ${ }^{(8,12-19)}$, prompting to a reverse causality bias. They also frequently focused on particular aspects of eating behaviours, such as food neophobia and pickiness.

In order to compare results across different populations, and to evaluate these relationships from a prospective approach, the objective of this study was to relate eating behaviours at different age ranges (4-6, 12-15, 24 and 48-54 months) with fruit and vegetable (F\&V) intake and dietary variety at $4-5$ years of age in three European birth cohorts (Generation XXI from Portugal, ALSPAC from the UK and EDEN from France). Our hypothesis was that problematic eating behaviours during early childhood may be associated with poorer dietary habits, and comparing results across different populations would give consistency to these findings.

\section{Methods}

\section{Description of cohorts}

Analyses were based on three European population-based birth cohorts: the Portuguese Generation XXI birth cohort, the British Avon Longitudinal Study of Parents and Children (ALSPAC) and the French EDEN mother-child cohort.

Generation XXI is a birth cohort that recruited women from all public maternity units from Porto, Portugal, between April 2005 and August 2006. A total of 8647 children and 8495 mothers were enrolled at baseline. Of the invited mothers, $91.4 \%$ agreed to participate ${ }^{(20)}$. Eating behaviours and feeding practices were assessed during intermediate follow-ups conducted in sub-samples at 6 and 15 months of age, and at 4 years of age, when $86 \%$ of all the children were re-evaluated (70\% by face-to-face interviews). For the present analyses, 912 children with complete data were included at 6 months, 544 at 15 months and 4227 at 48 months of age (see online Supplementary Table S1).

ALSPAC is a longitudinal birth cohort study, which recruited pregnant women resident in a geographically defined area in the south-west of England with an expected delivery date between April 1991 and December 1992. A cohort of 14541 pregnant women was established, resulting in 13988 children alive at 12 months of age. More details can be found on the ALSPAC website (http://www.bristol.ac.uk/alspac/). Eating behaviours and feeding practices were assessed by questionnaires sent to the main caregiver when the child was 6,15 and 54 months of age. For the present analyses, 5239 children with complete data were included at 6 months, 7685 at 15 months, 7418 at 24 months and 7620 at 54 months of age (see online Supplementary Table S1).

The EDEN mother-child cohort is a longitudinal study, which recruited 2002 pregnant women from two French university hospitals, in Nancy and Poitiers. Maternal perception of the child's appetite was assessed at 4, 12 and 24 months of age, difficulty to feed the child was assessed at 24 and 48 months of age and the child's food neophobia was assessed at 24 and 48 months of age. For the present analyses, 1034 children with complete data for both eating behaviour in early childhood and diet at 5 years were included in the 4-month analyses, 1002 in the 12-month analyses, 953 in the 24-month analyses and 892 in the 48-month analyses (see online Supplementary Table S1).

In each cohort, the procedures were carried out in accordance with the local and national ethical standards.

Subjects with missing values on variables of interest (eating behaviours; F\&V intake; the variety score; maternal age, education level and smoking; breast-feeding duration; and the child's BMI at 48-60 months) were excluded from the analyses. In cohorts with twins, one of them was selected at random to be included in the analyses. The different steps of sample selection are presented in online Supplementary Table S1.

\section{Fruit and vegetable intake and the Healthy Plate Variety Score}

Dietary information was assessed using FFQ at, respectively, 48, 54 and 60 months of age in the Generation XXI, ALSPAC and EDEN cohorts. Each cohort used their own FFQ, which varied in the number of items and frequency categories investigated. Table 1 provides descriptive data for each of the FFQ used in children.

Table 1. Description of the FFQ used in children at $4-5$ years of age in each of the three European birth cohorts

\begin{tabular}{lcccccccccc}
\hline Cohort & $\begin{array}{c}\text { Number of food } \\
\text { items/groups }\end{array}$ & Starchy & Fruits & Vegetables & Dairy & $\begin{array}{c}\text { Meat, fish and } \\
\text { alternatives }\end{array}$ & $\begin{array}{c}\text { Number of response } \\
\text { options }\end{array}$ & $\begin{array}{c}\text { Minimum daily } \\
\text { servings }\end{array}$ & $\begin{array}{c}\text { Maximum daily } \\
\text { servings }\end{array}$ \\
\hline $\begin{array}{l}\text { Generation } \\
\text { XXI }\end{array}$ & 35 & 2 & 1 & 3 & 5 & 5 & 9 & Never & $\geq 4 / \mathrm{d}$ \\
$\begin{array}{l}\text { ALSPAC } \\
\text { EDEN }\end{array}$ & 42 & 10 & 3 & 11 & 3 & 18 & 5 & Never & $>1 / \mathrm{d}$ \\
\hline
\end{tabular}


The FFQ data were harmonised by grouping questions about similar foods together in the same way in each cohort. The definition of fruits was the same in all cohorts - that is, exclusive of jams/jellies and fruit juice - and the definition of vegetables was exclusive of legumes and potatoes, but included the vegetable soup (typical in Portugal). Common cut-offs for fruits and for vegetables were used across the three cohorts, whenever possible ( $>1 v . \leq 1$ serving/d for fruits and for vegetables). In Generation XXI, the number of children in the $\leq 1$ serving/d category of vegetables was very low $(5.4 \%)$, mainly due to the high daily consumption of the vegetable soup in Portugal. Therefore, in Generation XXI, the cut-off used for vegetable frequency was $>3 v . \leq 3$ servings/d.

To assure the dietary data validity, some procedures were undertaken in each cohort. In Generation XXI, for a sub-sample of 2373 children, FFQ data was compared with 3-d food records completed at 4 years of age. In order to assess the validity of the FFQ, Pearson's correlation coefficients were calculated for key food groups as measured by both methods. For those food groups most frequently consumed, weak-to-moderate correlations and fair-to-moderate agreement were observed. Significant positive moderate Pearson's correlations were found for vegetable soup $(r=0.54, \quad P<0.001)$, fruit $(r=0.42$, $P<0.001)$, milk $(r=0.46, P<0.001)$ and yoghurts $(r=0.48$, $P<0.001)$, and not-so-strong correlations were found for cheese $(r=0.35, P<0.001)$, fish $(r=0.27, P<0.001)$, vegetables eaten on a plate $(r=0.26, P<0 \cdot 001)$, eggs $(r=0.21, P<0.001)$ and non-carbonated sugar-sweetened beverages (iced tea: $r=0.29, P<0.001$; juices: $r=0.22, P<0.001)$.

The ALSPAC children's FFQ was a modified version of the mother's FFQ, which was based on an FFQ developed by Yarnell et al. ${ }^{(21)}$. The FFQ administered when the children were 38 months old has been compared with 3-d food records collected in a sub-sample of children at 41 months of age, and the results were very similar.

The EDEN FFQ for children was a shortened version of the mother's validated $\mathrm{FFQ}^{(22)}$, but no specific validation was made for children.

To assess dietary variety, we calculated the Healthy Plate Variety Score (HPVS), which is a modified version of the food variety index for toddlers developed by Cox et al. ${ }^{(23)}$. The HPVS developed here uses FFQ data and is based on the food groups and the number of servings as recommended in the food plate model (former pyramid model) according to healthy eating guidelines promoted by the US Departments of Agriculture and Health and Human Services ${ }^{(24)}$.

To calculate the HPVS, food items were allocated to one of the following five food groups: (1) starchy foods (including potatoes); (2) fruits; (3) vegetables; (4) meat, fish and alternatives; and (5) dairy foods. The purpose of the HPVS is to measure variety both within and between food groups - that is, it aims to capture variety between the different food groups established, but also to ensure that the child does not always eat the same food within each food group (e.g. it should vary between milk, yoghurts, cheese, etc.).

Thus, truncations were applied, as advised by Cox et al. ${ }^{(23)}$, to assure that a high intake of one food group cannot compensate mathematically for a low intake of another food group, and that within each food group variety is ensured. Within each food group, the contribution of a particular food item was truncated at $33 \%$. Foods within a food group that are similar (e.g. pasta and noodles) were grouped together and count as a single food, so that they do not contribute more than $33 \%$. Owing to limited questions in some of the FFQs about types of fruits and vegetables, it was not possible to assess variety within these two groups; the score instead reflects whether children ate the recommended number of servings. After the groupings and truncations were applied, the number of servings for each food group was totalled. Food group scores were calculated by dividing the total number of servings by the recommended number of servings per $\mathrm{d}$ for each food group (starchy foods $=7$, fruit $=2$, vegetables $=3$, meat, fish and alternatives $=2$ and dairy foods $=3$ ). To ensure variety between the food groups, each food group score was truncated at 1.00 (e.g. if a child ate eight different types of starchy food, this was divided by seven, thus giving a potential score of $1 \cdot 14$, which was truncated to 1.00).

Foods such as condiments, sweets/candy, herbs/spices, soft drinks, oil, butter/margarine and salty snack foods were excluded from the analysis ${ }^{(23)}$. In addition, we also excluded cakes, biscuits/cookies, puddings and fried potatoes.

The final HPVS score is the sum of scores of the five food groups and can range from $0 \cdot 0$ to 5.0. A higher HPVS represents higher variety between and within food groups and also higher dietary adequacy. The HPVS was dichotomised by the median score in each cohort (possible range variation 0.0-5.0): 3.9 in Generation XXI, 3.1 in ALSPAC and 2.6 in EDEN cohorts.

\section{Assessment of eating behaviours}

Eating behaviours were reported as the perception of the main caregiver (usually mothers) on eating problems of their child. Only similar aspects of eating behaviours available in at least two cohorts and food neophobia (in EDEN only) were considered for this analysis, resulting in four main domains: feeding difficulties, poor eating, food refusal (for Generation XXI and ALSPAC)/food neophobia (for EDEN) and irregular eating. The questions used in each cohort are described in Table 3 (a more detailed explanation for food refusal and food neophobia are described in the table's footnote).

Each eating behaviour was dichotomised into 'Yes' (a considered aspect of eating behaviour was reported) $v$. 'No' (reference category). In Generation XXI, to define food refusal at 48 months of age, a combined variable was created to summarise refusal to take milk, F\&V, soup or fish ('Yes' was considered if at least one was reported). In the EDEN cohort, to define food neophobia, an index was created based on the mean of three questions and then dichotomised into ' $\geq$ median' (more neophobic) $v$. '<median' (considered as reference category).

Specific age ranges were selected, in which comparable data between cohorts were available: 4-6 months, 12-15 months and 48-54 months of age (with data available for Generation XXI, EDEN and ALSPAC) and 24 months of age (with data available for EDEN and ALSPAC). 


\section{Statistics}

Associations of eating behaviours with F\&V intake and the HPVS were estimated by odds ratio and 95\% confidence intervals obtained from unconditional logistic regression models. Associations were estimated prospectively, except at 48-54 months of age for Generation XXI and ALSPAC, where the associations were assessed cross-sectionally.

Four models were used with the indicated covariates: (i) model 1 , including as confounders those characteristics significantly associated with eating behaviours and dietary outcomes under study in at least two cohorts: maternal age, maternal education (baseline assessments), maternal smoking during pregnancy and any breast-feeding duration; (ii) model 2, including all variables from model 1 plus the child's World Health Organization ${ }^{(25)}$ BMI $z$-score at 4-5 years of age (as a continuous variable); (iii) models 3 and 4, including all variables from models 1 and 2 plus age at introduction of fruits or vegetables, age at introduction of any food other than F\&V and maternal F\&V intake (assessed during

Table 2. Characteristics of mothers and children at $4-5$ years of age by cohort

(Counts and percentages, mean values and standard deviations) in models 2-4, final tables only present results from model 2, which was considered as the final model.

All the analyses were performed using STATA/SE (StataCorp) version 10.0 in Generation XXI; SPSS 19.0 (SPSS Inc.) in ALSPAC; SAS software version 9.2 (SAS Institute Inc.) in EDEN.

\section{Results}

Table 2 shows the characteristics of mothers and children at 4-5 years of age, by cohort. These data suggest a similar distribution of some key variables across cohorts; more than $70 \%$ of mothers were between 25 and 35 years of age, approximately $20 \%$ smoked during pregnancy and $50 \%$ of the children were boys. Maternal education and child's breast-feeding duration differed across cohorts, which could be explained by country-specific socio-cultural differences. Generation XXI had the highest F\&V intake: 84\% of the Portuguese children presented a daily intake of F\&V above three servings compared with $62 \%$ in ALSPAC and $20 \%$ in EDEN cohorts.

\begin{tabular}{|c|c|c|c|c|c|c|}
\hline & \multicolumn{2}{|c|}{ Generation XXI ( $n$ 4227) } & \multicolumn{2}{|c|}{ ALSPAC $(n 7620)$} & \multicolumn{2}{|c|}{ EDEN $(n$ 892) } \\
\hline & Count & $\%$ & Count & $\%$ & Count & $\%$ \\
\hline \multicolumn{7}{|l|}{ Maternal age (years) } \\
\hline$<25$ & 649 & 15 & 1372 & 18 & 108 & 12 \\
\hline $25-35$ & 3025 & 72 & 5638 & 74 & 677 & 76 \\
\hline$>35$ & 553 & 13 & 610 & 8 & 108 & 12 \\
\hline \multicolumn{7}{|l|}{ Maternal education } \\
\hline Low & 815 & 19 & 1905 & 25 & 209 & 23 \\
\hline Intermediate & 2113 & 50 & 2743 & 36 & 147 & 16 \\
\hline High & 1299 & 31 & 2972 & 39 & 536 & 60 \\
\hline \multicolumn{7}{|l|}{ Smoking during pregnancy } \\
\hline Non-smoker & 3371 & 80 & 5590 & 77 & 717 & 80 \\
\hline Ever smoker & 856 & 20 & 2030 & 23 & 175 & 20 \\
\hline \multicolumn{7}{|l|}{ Child's sex } \\
\hline Boys & 2166 & 51 & 3962 & 52 & 472 & 53 \\
\hline Girls & 2061 & 49 & 3658 & 48 & 420 & 47 \\
\hline \multicolumn{7}{|l|}{ Child's gestational age (weeks) } \\
\hline$<37$ & 336 & 8 & 457 & 6 & 52 & 6 \\
\hline$\geq 37$ & 3852 & 91 & 7163 & 94 & 840 & 94 \\
\hline \multicolumn{7}{|l|}{ Child's birth weight (g) } \\
\hline Mean & \multicolumn{2}{|c|}{3168} & \multicolumn{2}{|c|}{3416} & \multicolumn{2}{|c|}{3290} \\
\hline SD & \multicolumn{2}{|c|}{515} & \multicolumn{2}{|c|}{539} & \multicolumn{2}{|c|}{507} \\
\hline \multirow{2}{*}{\multicolumn{7}{|c|}{ Child's $z$-score BMI at $4-5$ years* }} \\
\hline Mean & \multirow{2}{*}{\multicolumn{2}{|c|}{$\begin{array}{l}0.60 \\
1.08\end{array}$}} & \multirow{2}{*}{\multicolumn{2}{|c|}{$\begin{array}{l}0.75 \\
0.99\end{array}$}} & & 0.05 \\
\hline SD & & & & & & \\
\hline \multicolumn{7}{|l|}{ Any breast-feeding duration } \\
\hline Never & \multirow[t]{2}{*}{642} & \multirow[t]{2}{*}{$15 \dagger$} & 1829 & 24 & 242 & 27 \\
\hline$<1$ month & & & 762 & 10 & 61 & 7 \\
\hline$\geq 1$ to $<3$ months & 527 & 12 & 991 & 13 & 173 & 19 \\
\hline$\geq 3$ to $<6$ months & 758 & 18 & 1295 & 17 & 227 & 26 \\
\hline$\geq 6$ months & 2300 & 54 & 2743 & 36 & 189 & 21 \\
\hline Fruit intake at $4-5$ years $(>1$ serving/d) & 2590 & 61 & 3575 & 49 & 373 & 42 \\
\hline Vegetable intake at $4-5$ years $(>1$ serving/d) $\ddagger$ & 1424 & 34 & 2396 & 33 & 354 & 40 \\
\hline Fruit and vegetable intake at $4-5$ years ( $>3$ servings $/ \mathrm{d}$ ) & 3542 & 84 & 4710 & 62 & 187 & 21 \\
\hline HPVS at $4-5$ years & & & & & & \\
\hline Median & & & & & & \\
\hline 25-75th percentile & & & & & & \\
\hline
\end{tabular}

HPVS, Healthy Plate Variety Score.

* Defined according to the WHO $z$-scores ${ }^{(25)}$

$\dagger$ In Generation XXI, this \% combines the sample size for never and less than once a month.

$\ddagger$ In Generation XXI, the cut-off point used was three servings per d instead of one serving per d due to a higher vegetable intake in this population. 
Table 3 presents the prevalence of the selected eating behaviours at the different ages and cohorts. The nonestablishment of a daily routine by the child and the need of stimulation to eat were the eating behaviours less frequently reported by parents. Poor eating, defined as the child eating small quantities of each meal or not eating enough in Generation XXI and ALSPAC, was frequent (varying from $25 \%$ at 4-6 months to $66 \%$ at 24 months of age). Food refusal was reported more frequently by parents of older children ( $65 \%$ in Generation XXI and $68 \%$ in ALSPAC at 48-54 months of age), compared with less than $35 \%$ at 4-6 months of age.

Tables 4-6 describe the associations between the eating behaviours at the different ages and fruit intake (Table 4), vegetable intake (Table 5 ) and dietary variety, as defined by the HPVS (Table 6).

Children with more eating difficulties, as reported by parents at the different ages, were less likely to have fruit intake $>1$ serving/d when compared with children with no eating difficulties (Table 4). This association was consistent across cohorts and ages. Similarly, children presenting refusal to foods or higher food neophobia scores and difficulties in establishing a daily routine, particularly at 24 and 48-60 months of age, had lower fruit intake at 4-5 years of age compared with children with no such eating difficulties. The association of poor eating with fruit intake was less consistent.

When compared with fruits, the results for vegetable intake were very similar, but slightly stronger; inverse associations were found between vegetable intake and eating difficulties namely, at early stages of life - and also poor eating, food refusal/neophobia and difficulties in establishing a daily routine, particularly when reported at 12 months of age and thereafter (Table 5).

Children whose parents reported these eating difficulties at $4-6,12-15$ and 24 months of age also presented a lower variety score at $4-5$ years of age than children with no eating difficulties (Table 6). The association with food refusal/neophobia and difficulties in establishing a daily routine were in the same direction, but only significant when eating behaviours were reported after 12 months of age.

\section{Discussion}

Feeding problems in infants and toddlers were frequently reported by mothers from the three European birth cohorts, and were shown to be prospectively associated with lower F\&V intake as well as with less dietary variety at $4-5$ years of age.

This study, based on population-based samples, has shown that the prevalence of feeding problems varied from 3 to $66 \%$. Previous studies have already described that feeding problems are common in children; approximately $20-50 \%$ of normally developing children are reported to experience some type of feeding problems ${ }^{(6,12,17,26,27)}$. Most of these feeding problems are likely to be transient, and because some evidence suggests that the nature and extent of feeding problems decline as a function of the age of the child ${ }^{(28)}$ the present study examined whether differences would be evident across development. We found that feeding problems seemed to be more prevalent at older ages and that the associations reported were also more 
Table 4. Association between eating behaviours and high fruit intake (>1 serving/d) at 48 months in Generation XXI, 54 months in ALSPAC and 60 months in EDEN (Odds ratios and $95 \%$ confidence intervals)

\begin{tabular}{|c|c|c|c|c|c|c|c|c|c|}
\hline \multirow[b]{2}{*}{ Yes $v$. no (reference) $\dagger$} & \multicolumn{2}{|c|}{ Feeding difficulties } & \multicolumn{2}{|c|}{ Poor eating $\ddagger$} & \multicolumn{2}{|c|}{ Food refusal/food neophobia§ } & \multicolumn{3}{|c|}{ Difficulties in establishing a daily routine } \\
\hline & OR & $95 \% \mathrm{Cl}$ & OR & $95 \% \mathrm{Cl}$ & OR & $95 \% \mathrm{Cl}$ & OR & & $95 \% \mathrm{Cl}$ \\
\hline \multicolumn{10}{|l|}{ 4-6 months } \\
\hline Generation XXI & 0.74 & $0.50,1.09$ & 1.13 & $0.82,1.56$ & 0.87 & $0.64,1.19$ & 0.78 & & $0.48,1.26$ \\
\hline ALSPAC & $0.82^{\star}$ & $0.72,0.93$ & 1.07 & $0.94,1.22$ & 1.07 & $0.92,1.26$ & 0.96 & & $0.80,1.15$ \\
\hline EDEN & & $\mathrm{N} / \mathrm{A}$ & 1.40 & $0.67,2.93$ & \multicolumn{2}{|c|}{$\mathrm{N} / \mathrm{A}$} & \multicolumn{3}{|r|}{$0.00,1.15$} \\
\hline \multicolumn{10}{|l|}{$12-15$ months } \\
\hline Generation XXI & $0.69^{*}$ & $0.48,0.99$ & $0.63^{*}$ & $0.44,0.92$ & 0.72 & $0.50,1.04$ & 0.97 & & $0.57,1.66$ \\
\hline ALSPAC & $0.77^{*}$ & $0.67,0.88$ & 1.03 & $0.90,1.17$ & 0.89 & $0.78,1.01$ & 0.86 & & $0.71,1.03$ \\
\hline EDEN & \multicolumn{2}{|r|}{ N/A } & 0.82 & $0.44,1.48$ & \multicolumn{2}{|c|}{$\mathrm{N} / \mathrm{A}$} & \multicolumn{3}{|c|}{$N / A$} \\
\hline \multicolumn{10}{|l|}{24 months } \\
\hline ALSPAC & $0.76^{*}$ & $0.67,0.86$ & $0.87^{\star}$ & $0.76,0.99$ & $0.84^{*}$ & $0.72,0.98$ & \multirow{2}{*}{\multicolumn{3}{|c|}{$0.72,0.98$}} \\
\hline EDEN & $0.67^{*}$ & $0.49,0.93$ & 1.12 & $0.63,1.98$ & $0.60^{*}$ & $0.46,0.79$ & & & \\
\hline \multicolumn{10}{|l|}{$48-60$ months } \\
\hline Generation XXI & $0.66^{*}$ & $0.58,0.76$ & $0.71^{*}$ & $0.62,0.81$ & $0.58^{*}$ & $0.51,0.67$ & $0.53^{*}$ & & $0.41,0.67$ \\
\hline ALSPAC & $0.75^{\star}$ & $0.67,0.84$ & $0.80^{\star}$ & $0.71,0.91$ & $0.85^{\star}$ & $0.76,0.96$ & $0.77^{\star}$ & & $0.67,0.88$ \\
\hline EDEN & 0.72 & $0.51,1.02$ & & $\mathrm{~N} / \mathrm{A}$ & $0.62^{*}$ & $0.47,0.82$ & \multicolumn{3}{|c|}{$\mathrm{N} / \mathrm{A}$} \\
\hline
\end{tabular}

$\mathrm{N} / \mathrm{A}$, eating behaviour not available at that age.

${ }^{*} P<0.05$.

† OR and respective $95 \% \mathrm{Cl}$ adjusted for maternal age, education, smoking during pregnancy, any breast-feeding and child's $z$-score BMI.

‡ Poor eating corresponding to eats small quantities at each meal (at 4-6 months), does not eat enough (at 12-15 months) in ALSPAC and Generation XXI and needs to be stimulated in EDEN (at 4, 12 and 24 months).

§ Refusal to milk at 4-6 months and solids at 12-15 months in Generation XXI, refusal to milk at 4-6 months and to the right food at 12-15 months and 24 months in ALSPAC, refusal to the right food at 48-54 months in Generation XXI and ALSPAC. Food neophobia score available only in EDEN and calculated based on the mean of three questions ( $\geq$ median (more neophobic) $v$. < median (reference category)).

Table 5. Association between eating behaviours and high vegetable intake ( $>1$ serving/d in ALSPAC and EDEN and $>3$ servings/d in Generation XXI) at 48 months in Generation XXI, 54 months in ALSPAC and 60 months in EDEN

(Odds ratios and $95 \%$ confidence intervals)

\begin{tabular}{|c|c|c|c|c|c|c|c|c|c|}
\hline \multirow[b]{2}{*}{ Yes $v$. no (reference) $\dagger$} & \multicolumn{2}{|c|}{ Feeding difficulties } & \multicolumn{2}{|c|}{ Poor eating $\ddagger$} & \multicolumn{2}{|c|}{ Food refusal/food neophobia§ } & \multicolumn{3}{|c|}{ Difficulties in establishing a daily routine } \\
\hline & OR & $95 \% \mathrm{Cl}$ & OR & $95 \% \mathrm{Cl}$ & OR & $95 \% \mathrm{Cl}$ & OR & & $95 \% \mathrm{Cl}$ \\
\hline \multicolumn{10}{|l|}{ 4-6 months } \\
\hline Generation XXI & $0.65^{*}$ & $0.43,0.97$ & 1.00 & $0.73,1.37$ & 1.04 & $0.76,1.41$ & 0.78 & & $0.47,1.28$ \\
\hline ALSPAC & $0.85^{*}$ & $0.74,0.97$ & 1.04 & $0.90,1.20$ & 1.08 & $0.91,1 \cdot 28$ & 0.84 & & $0.70,1.01$ \\
\hline EDEN & \multicolumn{2}{|r|}{$N / A$} & 0.53 & $0.22,1.22$ & \multicolumn{2}{|c|}{$\mathrm{N} / \mathrm{A}$} & \multicolumn{3}{|c|}{ N/A } \\
\hline \multicolumn{10}{|l|}{$12-15$ months } \\
\hline Generation XXI & 1.08 & $0.74,1.58$ & $1.07^{*}$ & $0.73,1.56$ & $0.67^{*}$ & $0.45,0.98$ & 1.08 & & $0.62,1.89$ \\
\hline ALSPAC & 0.90 & $0.78,1.04$ & 1.13 & $0.98,1.30$ & $0.73^{*}$ & $0.63,0.84$ & 0.84 & & $0.69,1.03$ \\
\hline EDEN & \multicolumn{2}{|r|}{ N/A } & 1.02 & $0.56,1.87$ & \multicolumn{2}{|c|}{ N/A } & \multicolumn{3}{|c|}{ N/A } \\
\hline \multicolumn{10}{|l|}{24 months } \\
\hline ALSPAC & $0.56^{*}$ & $0.49,0.64$ & $0.67^{*}$ & $0.58,0.78$ & $0.50^{\star}$ & $0.44,0.58$ & \multirow{2}{*}{\multicolumn{3}{|c|}{$0.40,0.00$}} \\
\hline EDEN & $0.69^{*}$ & $0.49,0.97$ & 1.16 & $0.64,2 \cdot 10$ & $0.61^{*}$ & $0.46,0.80$ & & & \\
\hline \multicolumn{10}{|l|}{$48-60$ months } \\
\hline Generation XXI & $0.64^{*}$ & $0.56,0.73$ & $0.69^{*}$ & $0.60,0.80$ & $0.38^{*}$ & $0.33,0.43$ & $0.48^{\star}$ & & $0.36,0.66$ \\
\hline ALSPAC & $0.33^{*}$ & $0.29,0.38$ & $0.59^{*}$ & $0.52,0.67$ & $0.38^{*}$ & $0.33,0.44$ & $0.43^{*}$ & & $0.38,0.50$ \\
\hline EDEN & $0.50^{*}$ & $0.34,0.73$ & & $\mathrm{~N} / \mathrm{A}$ & $0.57^{*}$ & $0.43,0.76$ & \multicolumn{3}{|c|}{ N/A } \\
\hline
\end{tabular}

$\mathrm{N} / \mathrm{A}$, eating behaviour not available at that age.

${ }^{*} P<0.05$.

† OR and respective $95 \% \mathrm{Cl}$ adjusted for maternal age, education, smoking during pregnancy, any breast-feeding and child's $z$-score BMI.

‡ Poor eating corresponding to eats small quantities at each meal (at 4-6 months), does not eat enough (at 12-15 months) in ALSPAC and Generation XXI and needs to be stimulated in EDEN (at 4, 12 and 24 months).

§ Refusal to milk at 4-6 months and solids at 12-15 months in Generation XXI, refusal to milk at 4-6 months and to the right food at 12-15 months and 24 months in ALSPAC, refusal to the right food at 48-54 months in Generation XXI and ALSPAC. Food neophobia score available only in EDEN and calculated based on the mean of three questions ( $\geq$ median (more neophobic) $v$. < median (reference category)).

evident at older ages (i.e. after 12 months of age). As previously shown in a prospective study of 120 children and their parents, followed-up from 2 to 11 years of age ${ }^{(5)}$, incidence of picky eating declined over time, whereas point prevalence increased indicating that picky eating is often a chronic problem with $40 \%$ having a duration of more than 2 years of age.

In fact, feeding problems in infants appear to be highly persistent throughout childhood ${ }^{(29)}$, and the persistence of these eating behaviours over time can have long-term effects that are not evident until children are older; they might be precursors of unhealthy eating and may worsen their weight status $^{(7,8)}$ later in life, which highlights the need of prospective analysis. In the present study, associations were tested prospectively, except at 4-5 years of age, in Generation XXI and ALSPAC when eating behaviours were reported at the same time than dietary intake. 
Table 6. Association between eating behaviours and a higher score in the Healthy Plate Variety Score (>median in the three cohorts) at 48 months in Generation XXI, 54 months in ALSPAC and 60 months in EDEN

(Odds ratios and $95 \%$ confidence intervals)

\begin{tabular}{|c|c|c|c|c|c|c|c|c|c|}
\hline \multirow[b]{2}{*}{ Yes v. No (reference) $\dagger$} & \multicolumn{2}{|c|}{ Feeding difficulties } & \multicolumn{2}{|c|}{ Poor eating $\ddagger$} & \multicolumn{2}{|c|}{ Food refusal/food neophobia§ } & \multicolumn{3}{|c|}{ Difficulties in establishing a daily routine } \\
\hline & OR & $95 \% \mathrm{Cl}$ & OR & $95 \% \mathrm{Cl}$ & OR & $95 \% \mathrm{Cl}$ & OR & & $95 \% \mathrm{Cl}$ \\
\hline \multicolumn{10}{|l|}{ 4-6 months } \\
\hline Generation XXI & $0.67^{\star}$ & $0.45,0.98$ & $1 \cdot 28$ & $0.94,1.74$ & 1.16 & $0.85,1.57$ & 0.76 & & $0.46,1.25$ \\
\hline ALSPAC & $0.86^{*}$ & $0.75,0.98$ & 0.96 & $0.84,1.09$ & 0.98 & $0.84,1.15$ & 1.01 & & $0 \cdot 85,1 \cdot 21$ \\
\hline EDEN & & $N / A$ & 0.69 & $0.32,1.48$ & \multicolumn{2}{|c|}{$\mathrm{N} / \mathrm{A}$} & \multicolumn{3}{|c|}{ N/A } \\
\hline \multicolumn{10}{|l|}{$12-15$ months } \\
\hline Generation XXI & 0.82 & $0.55,1.22$ & $0.61^{*}$ & $0.41,0.91$ & $0.55^{\star}$ & $0.36,0.84$ & 1.17 & & $0 \cdot 6,2 \cdot 10$ \\
\hline ALSPAC & $0.66^{*}$ & $0.58,0.76$ & 1.00 & $0.88,1.15$ & $1 \cdot 20^{*}$ & $1.05,1.37$ & $0.76^{*}$ & & $0.63,0.92$ \\
\hline EDEN & \multicolumn{2}{|r|}{$\mathrm{N} / \mathrm{A}$} & 0.71 & $0 \cdot 39,1 \cdot 29$ & \multicolumn{2}{|c|}{ N/A } & \multicolumn{3}{|c|}{$\mathrm{N} / \mathrm{A}$} \\
\hline \multicolumn{10}{|l|}{24 months } \\
\hline ALSPAC & $0.61^{*}$ & $0.54,0.70$ & $0.80^{*}$ & $0.70,0.91$ & $0.67^{\star}$ & $0.59,0.77$ & $0.64^{*}$ & & $0.5,0.75$ \\
\hline EDEN & $0.45^{\star}$ & $0.32,0.63$ & 1.32 & $0.74,2.35$ & $0.57^{\star}$ & $0.44,0.74$ & \multicolumn{3}{|r|}{ של ס של } \\
\hline \multicolumn{10}{|l|}{$48-60$ months } \\
\hline Generation XXI & $0.56^{\star}$ & $0.49,0.65$ & $0.67^{\star}$ & $0.58,0.76$ & $0.44^{*}$ & $0.39,0.51$ & $0.43^{*}$ & & $0.32,0.57$ \\
\hline ALSPAC & $0.55^{\star}$ & $0.49,0.62$ & $0.76^{*}$ & $0.67,0.85$ & $0.65^{\star}$ & $0.58,0.74$ & $0.55^{\star}$ & & $0.48,0.63$ \\
\hline EDEN & $0.49^{*}$ & $0.34,0.69$ & & N/A & $0.53^{*}$ & $0.40,0.70$ & \multicolumn{3}{|c|}{$\mathrm{N} / \mathrm{A}$} \\
\hline
\end{tabular}

$\mathrm{N} / \mathrm{A}$, eating behaviour not available at that age.

* $P<0.05$.

† OR and respective $95 \% \mathrm{Cl}$ adjusted for maternal age, education, smoking during pregnancy, any breast-feeding and child's z-score BMI.

‡ Poor eating corresponding to eats small quantities at each meal (at 4-6 months), does not eat enough (at 12-15 months) in ALSPAC and Generation XXI and needs to be stimulated in EDEN (at 4, 12 and 24 months).

§ Refusal to milk at 4-6 months and solids at 12-15 months in Generation XXI, refusal to milk at 4-6 months and to the right food at 12-15 months and 24 months in ALSPAC, refusal to the right food at 48-54 months in Generation XXI and ALSPAC. Food neophobia score available only in EDEN and calculated based on the mean of three questions ( $\geq$ median (more neophobic) $v$. $<$ median (reference category)).

Most studies conducted so far linking feeding problems with dietary intake ${ }^{(7,8,12-17)}$ were, however, cross-sectional ${ }^{(8,12-17)}$. They suggest that neophobia and pickiness are both associated with less healthy food choices in children. In the study by Galloway et $a l^{(8)}$, 9-year-old girls who were picky eaters consumed significantly fewer servings of fruits, vegetables, fats and sweets as well as less fibre than girls who were not picky eaters. These results suggest that picky eaters did not consume more fats and sweets to compensate for lower F\&V intake. In a naturalistic mealtime situation, Cooke et al. ${ }^{(13)}$ have also concluded in children aged 4-5 years that food neophobia impacts differentially on consumption of different food types. Specifically, it appears that children who score highly on the Child Food Neophobia Scale eat less fruit, vegetables and protein foods than their less neophobic peers.

Other studies have also attempted to investigate the impact of neophobia on dietary variety ${ }^{(5,18,19)}$. Falciglia et $a l^{(19)}$ in 9- to 10-year-old children found that the Healthy Eating Index scores were lower for the neophobic group than for the other children. Carruth et $a l{ }^{(18)}$ also showed that toddlers perceived by their mothers as picky eaters had significantly lower dietary variety and diversity scores. Mascola et al. ${ }^{(5)}$ reported that parents of picky eaters were more likely to report that their children consumed a limited variety of foods, evaluated through a single question of the Stanford Feeding Questionnaire.

In a Canadian study, mothers' perception of children's eating behaviours was prospectively linked to higher body weight and less dietary adequacy at 4.5 years of age ${ }^{(7)}$. This study indicated that children with picky eating at $2.5,3.5$ and 4.5 years of age are under-consuming - at 4.5 years - some macronutrients (less fat, protein and total energy) and food groups, such as F\&V and meat and alternatives, whereas overeaters are over-consuming grain products and meat and alternatives. To our knowledge, this is the only study presenting prospective data, but dietary adequacy was evaluated by comparison with the national recommendations - Canada's Food Guide - and no summary measure of adequacy was provided. Nonetheless, data suggest that picky eaters have less dietary variety. Our data enlarged these findings, supporting that other eating behaviours such as food refusal/neophobia and difficulties in establishing a daily routine perceived at early ages are prospectively associated with lower F\&V intake and less dietary variety, measured by a composite score that takes into account variety within and across food groups, when children were $4-5$ years old. Our data also showed that the prospective and cross-sectional associations were quite comparable in magnitude after 12 months, which is again in favour of an actual association.

In the present study, the associations of eating behaviours with fruits and vegetables were in the same direction compared with dietary variety. This is in agreement with the notion that less varied diets have also lower intake of more healthy foods, such as F\&V, and higher intake of unhealthy foods ${ }^{(30)}$. Moreover, the inverse associations of these eating behaviours were slightly stronger with vegetables than with fruits. Cooke et al. ${ }^{(15)}$ have already described that food neophobia is more strongly correlated with vegetables than with fruit intake. Vegetables are bitter and infants are born with an innate liking for sweet tastes and a dislike of sour or bitter ones ${ }^{(31)}$, thus they have more difficulties in accepting bitter-tasting vegetables, for which they have to learn to accept them. In addition, when children's vegetable consumption was explored in more detail, the sweeter vegetables tend to be preferred ${ }^{(32)}$.

Feeding problems, such as those reported in this study, are liable to have a strong behavioural aetiology ${ }^{(2)}$, thus they can be overcome. There is good evidence that modelling and repeated exposure are strategies that enhance acceptance of a variety of 
foods ${ }^{(33-35)}$. Therefore, as suggested by Cooke et al., 'guiding parents in the technique of regular and repeated taste exposure has the potential to improve diet's quality of young children at what may be a sensitive period for developing lifelong healthy eating patterns, ${ }^{,(15)}$.

Definitions for eating behaviours vary across studies. Our findings highlight a lack of clarity that persists concerning classification of children's problematic eating behaviours and the need of better instruments with which to measure them. Even in the present study, for some aspects of eating behaviours (especially for poor eating and food refusal), the questions differed between the ages and cohorts. However, consistent associations were reported, suggesting that these methodological differences would not influence our results.

This study has used the data collected from three different European countries in parallel analyses using the same confounders, and an attempt at data harmonisation was made. Each cohort used their own FFQ, which varied in the number of items and frequency categories; however, the core foods that most children's diets consist of were covered. To ensure comparability between the countries, the daily number of servings was calculated in each country and, whenever possible, the same cut-off points were used. This was not possible in Generation XXI concerning vegetables, as there is a much higher frequency of vegetable consumption in Portugal than in France and the UK, as previously shown ${ }^{(36)}$. In addition, the dietary variety score was calculated in the same way in each country. Although we have assumed that the dietary variety score is a marker for diet quality, we are unable to validate this hypothesis, yet the foods recommended in the healthy plate model are based on guidelines for healthy eating and should reflect a diet of high quality.

In the present study, bias attributable to selective loss to follow-up cannot be discounted and may have weakened our results. In addition, in some age ranges and cohorts, the effect sizes were small and the CI large; however, the comparisons performed between different cohorts and age ranges provide more consistency to our results, and again the consistent results found across cohorts suggest a low likelihood of bias.

\section{Conclusions}

Children with eating difficulties, food refusal/neophobia and difficulties in establishing a daily routine, as reported by their mothers, presented lower F\&V intake and less dietary variety at 4-5 years of age. These associations were consistent across three European birth cohorts and more evident when eating behaviours were reported after 12 months of age, and were slightly stronger for vegetables than fruits.

Efforts to improve dietary quality in early childhood could incorporate strategies aimed at reducing these problematic eating behaviours, such as modelling and taste exposure ${ }^{(33-35)}$.

\section{Acknowledgements}

We are grateful to Sylvie Issanchou for her coordination of the HabEat project. We are indebted to all participants for providing the data used in the three birth cohorts, as well as to all the members of the research team and coordinators (Henrique Barros from Generation XXI; George Davey Smith from ALSPAC and Barbara Heude from the EDEN Mother-Child Study Group).

This study was supported by the European Community's Seventh Framework Program (FP7/2007-2013) under the grant agreement no. FP7-245012-HabEat. Generation XXI was funded by Programa Operacional de Saúde - Saúde XXI, Quadro Comunitário de Apoio III and by Administração Regional de Saúde Norte. For follow-up assessments Generation XXI received funding from Fundação para a Ciência e a Tecnologia, co-funded by FEDER through COMPETE and from Fundação Calouste Gulbenkian. The UK Medical Research Council (grant ref: 74882), the Wellcome Trust (grant ref: 076467) and the University of Bristol provided core support for ALSPAC. Support for the EDEN (Etude des Déterminants pre et postnatals précoces du développement et de la santé de l'ENfant) Study was provided by the Foundation for Medical Research, the French Ministry of Research (IFR programme), the Institut National de la Santé et de la Recherche Médicale (Human Nutrition Research Program), the Diabetes National Research Program (via a collaboration with the French Association for Diabetes Research), the French Ministry of Health Perinatality Program, the French Agency for Environmental Security, the French National Institute for Population Health Surveillance, the Paris-Sud University, the French National Institute for Health Education, Nestlé, the National Education Health Insurance, the French Speaking Association for the Study of Diabetes and Metabolism, the National Agency for Research (non-thematic programme) and the National Institute for Research in Public Health (TGIR health cohort 2008 programme). Study sponsors were not involved in the study design, data collection or data analyses.

Study concept and design: O. A, J. L., d. L.-G. B., E. P., M. P., C. M. A. and L. C. Acquisition of data: O. A., J. L., d. L.-G. B., E. P., M. P., C. M. A. and L. C. Analysis of data: O. A., J. L. and d. L.-G. B. Drafting of the manuscript: O. A. Critical revision of the manuscript and final approval: O. A., J. L., d. L.-G. B., E. P., M. P., C. M. A. and L. C.

There are no conflicts of interest.

\section{Supplementary Material}

For supplementary material/s referred to in this article, please visit http://dx.doi.org/10.1017/S0007114515002287

\section{References}

1. Bernard-Bonnin AC (2006) Feeding problems of infants and toddlers. Can Fam Physician 52, 1247-1251.

2. Aldridge VK, Dovey TM, Martin CI, et al. (2010) Identifying clinically relevant feeding problems and disorders. I Child Health Care 14, 261-270.

3. Skinner J, Carruth BR, Houck K, et al. (1998) Mealtime communication patterns of infants from 2 to 24 months of age. J Nutr Educ 30, 8-16.

4. Dovey TM, Staples PA, Gibson EL, et al. (2008) Food neophobia and 'picky/fussy' eating in children: a review. Appetite 50, 181-193. 
5. Mascola AJ, Bryson SW \& Agras WS (2010) Picky eating during childhood: a longitudinal study to age 11 years. Eat Behav 11, 253-257.

6. Reau NR, Senturia YD, Lebailly SA, et al. (1996) Infant and toddler feeding patterns and problems: normative data and a new direction. Pediatric Practice Research Group. J Dev Behav Pediatr 17, 149-153.

7. Dubois L, Farmer AP, Girard M, et al. (2007) Preschool children's eating behaviours are related to dietary adequacy and body weight. Eur J Clin Nutr 61, 846-855.

8. Galloway AT, Fiorito L, Lee Y, et al. (2005) Parental pressure, dietary patterns, and weight status among girls who are "picky eaters". J Am Diet Assoc 105, 541-548.

9. Jacobi C, Schmitz G \& Agras WS (2008) Is picky eating an eating disorder? Int J Eat Disord 41, 626-634.

10. Winsper C \& Wolke D (2013) Infant and toddler crying, sleeping and feeding problems and trajectories of dysregulated behavior across childhood. J Abnorm Child Psychol 42, 831-843.

11. Marchi M \& Cohen P (1990) Early childhood eating behaviors and adolescent eating disorders. J Am Acad Child Adolesc Psychiatry 29, 112-117.

12. Carruth BR, Ziegler PJ, Gordon A, et al. (2004) Prevalence of picky eaters among infants and toddlers and their caregivers' decisions about offering a new food. J Am Diet Assoc 104, s57-s64.

13. Cooke L, Carnell S \& Wardle J (2006) Food neophobia and mealtime food consumption in $4-5$ year old children. Int J Behav Nutr Phys Act 3, 14.

14. Galloway AT, Lee Y \& Birch LL (2003) Predictors and consequences of food neophobia and pickiness in young girls. J Am Diet Assoc 103, 692-698.

15. Cooke L, Wardle J \& Gibson EL (2003) Relationship between parental report of food neophobia and everyday food consumption in 2-6-year-old children. Appetite 41, 205-206.

16. Cooke LJ, Wardle J, Gibson EL, et al. (2004) Demographic, familial and trait predictors of fruit and vegetable consumption by pre-school children. Public Health Nutr 7, 295-302.

17. Jacobi C, Agras WS, Bryson S, et al. (2003) Behavioral validation, precursors, and concomitants of picky eating in childhood. J Am Acad Child Adolesc Psychiatry 42, 76-84.

18. Carruth BR, Skinner J, Houck K, et al. (1998) The phenomenon of 'picky eater': a behavioral marker in eating patterns of toddlers. J Am Coll Nutr 17, 180-186.

19. Falciglia GA, Couch SC, Gribble LS, et al. (2000) Food neophobia in childhood affects dietary variety. $J$ Am Diet Assoc 100, 1474-1481.

20. Larsen PS, Kamper-Jorgensen M, Adamson A, et al. (2013) Pregnancy and birth cohort resources in Europe: a large opportunity for aetiological child health research. Paediatr Perinat Epidemiol 27, 393-414.
21. Yarnell JW, Fehily AM, Milbank JE, et al. (1983) A short dietary questionnaire for use in an epidemiological survey: comparison with weighed dietary records. Hum Nutr Appl Nutr 37, 103-112.

22. Deschamps V, de Lauzon-Guillain B, Lafay L et al. (2009) Reproducibility and relative validity of a food-frequency questionnaire among French adults and adolescents. Eur J Clin Nutr 63, 282-291.

23. Cox DR, Skinner JD, Carruth BR, et al. (1997) A Food Variety Index for Toddlers (VIT): development and application. J Am Diet Assoc 97, 1387-1388 (quiz 1382-1386).

24. US Departments of Agriculture and Health and Human Services (2010) Dietary Guidelines for Americans, 7th ed. Washington, DC: Government Printing Office.

25. World Health Organization (2006) WHO Child Growth Standards based on length/height, weight and age. Acta Paediatr Suppl 450, 76-85.

26. Benjasuwantep $B$, Chaithirayanon $S$ \& Eiamudomkan $M$ (2013) Feeding problems in healthy young children: prevalence, related factors and feeding practices. Pediatr Rep 5, 38-42.

27. Wright CM, Parkinson KN, Shipton D, et al. (2007) How do toddler eating problems relate to their eating behavior, food preferences, and growth? Pediatrics 120, e1069-e1075.

28. Koivisto UK \& Sjoden PO (1996) Food and general neophobia in Swedish families: parent-child comparisons and relationships with serving specific foods. Appetite 26, 107-118.

29. Dahl M, Rydell AM \& Sundelin C (1994) Children with early refusal to eat: follow-up during primary school. Acta Paediatr 83, 54-58.

30. Murphy SP, Foote JA, Wilkens LR, et al. (2006) Simple measures of dietary variety are associated with improved dietary quality. J Am Diet Assoc 106, 425-429.

31. Steiner JE (1974) Discussion paper: innate, discriminative human facial expressions to taste and smell stimulation. Ann NY Acad Sci 237, 229-233.

32. Ahern SM, Caton SJ, Bouhlal S, et al. (2013) Eating a rainbow. Introducing vegetables in the first years of life in 3 European countries. Appetite 71, 48-56.

33. Birch LL \& Marlin DW (1982) I don't like it; I never tried it: effects of exposure on two-year-old children's food preferences. Appetite 3, 353-360.

34. Birch LL (1980) Effects of peer models' food choices and eating behaviors on preschoolers' food preferences. Child Dev 51, 489-496.

35. Cooke L (2007) The importance of exposure for healthy eating in childhood: a review. J Hum Nutr Diet 20, 294-301.

36. de Lauzon-Guillain B, Jones L, Oliveira A, et al. (2013) The influence of early feeding practices on fruit and vegetable intake among preschool children in 4 European birth cohorts. Am J Clin Nutr 98, 804-812. 